\title{
Germinação de sementes de espécies de Melastomataceae de Cerrado sob condições controladas de luz e temperatura
}

\author{
Rosana Cristina Carreira ${ }^{1,2}$ e Lilian Beatriz Penteado Zaidan ${ }^{1}$
}

Recebido: 03.08.2006; aceito: 18.06.2007

ABSTRACT - (Germination of seeds of Melastomataceae species from the Cerrado under controlled light and temperature). The aim of this study was to investigate the germination of seeds of Miconia albicans, M. langosdorffii, M. rubiginosa, M. stenostachya, Schizocetron elegans and Tibouchina gracilis that occur in Cerrado areas. The seeds were exposed daily to light during 0, $10 \mathrm{~min}, 15 \mathrm{~min}, 30 \mathrm{~min}, 60 \mathrm{~min}, 90 \mathrm{~min}, 180 \mathrm{~min}, 360 \mathrm{~min}, 720 \mathrm{~min}$ and $1.440 \mathrm{~min}$ at $25^{\circ} \mathrm{C}$. Five gradients of phytochrome photoequilibrium (j) were tested in seeds of M. albicans and M. rubiginosa. The effect of temperature was observed at constant $15,20,25,30$ and $35^{\circ} \mathrm{C}$, and alternated temperatures of $30 / 20^{\circ} \mathrm{C}$, under continuous light and darkness. Germinability and speed of germination were analyzed. Seeds of all the species are photoblastic. Seeds of M. albicans, M. langsdorffii, S. elegans and T. gracilis need at least 60 to $90 \mathrm{~min}$ of light to start germination. $M$. stenostachya $(15 \mathrm{~min})$ and $M$. rubiginosa $(360 \mathrm{~min}$ ) showed the extreme responses to light treatment. The germinability of the seeds of M. albicans increased under higher gradients of R:FR photoequilibrium. Germination was low in M. rubiginosa, so the response of the seeds to R:FR photoequilibrium was inconclusive. In relation to temperature, germination occurred mainly between constant $20^{\circ} \mathrm{C}$ and $30^{\circ} \mathrm{C}$ and at $30 / 20^{\circ} \mathrm{C}$. Germination under light and with temperatures between $20^{\circ} \mathrm{C}$ and $30^{\circ} \mathrm{C}$ are characteristics that may contribute to the establishment of plantlets of the Melastomataceae in the cerrado. The seeds remain in the soil seed bank and may germinate when light is available even for short periods and despite the daily variation in temperature.

Key words: light, Melastomataceae, seed germination, temperature

RESUMO - (Germinação de sementes de espécies de Melastomataceae de cerrado sob condições controladas de luz e temperatura). Foram investigados os efeitos de luz e temperatura na germinação de sementes de Miconia albicans, M. langosdorffii, M. rubiginosa, M. stenostachya, Schizocetron elegans e Tibouchina gracilis (Melastomataceae). As sementes foram expostas diariamente à luz por $0,10 \mathrm{~min}, 15 \mathrm{~min}, 30 \mathrm{~min}, 60 \mathrm{~min}, 90 \mathrm{~min}, 180 \mathrm{~min}, 360 \mathrm{~min}, 720 \mathrm{~min}$ and 1.440 min a $25^{\circ} \mathrm{C}$. Foram fornecidos cinco gradientes de fotoequilíbrio do fitocromo (j) às sementes de $M$. albicans e $M$. rubiginosa. A germinação das sementes foi testada a $15,20,25,30$ e $35^{\circ} \mathrm{C}$ e a $30 / 20{ }^{\circ} \mathrm{C}$, sob luz e escuro contínuos. As medidas de germinação analisadas foram germinabilidade, tempo e velocidade médios de germinação. As sementes das espécies analisadas são fotoblásticas. M. albicans, M. langsdorffii, S. elegans e T. gracilis exigem entre 60 e 90 min diários de luz para desencadear o processo de germinação. M. stenostachya (15 min) e M. rubiginosa (360 min) seriam os extremos desse tipo de comportamento. A germinabilidade das sementes de M. albicans aumentou sob gradientes crescentes de V:VE. As sementes de M. rubiginosa tiveram baixa germinabilidade, sendo inconclusiva sua resposta a esse tratamento. Com relação à temperatura, a germinação das sementes ocorreu preferencialmente entre 20 e $30{ }^{\circ} \mathrm{C}$ e na temperatura alternada. Germinação na luz e na faixa de temperatura entre $20^{\circ} \mathrm{C}$ e $30{ }^{\circ} \mathrm{C}$ são características que contribuem para $\mathrm{o}$ sucesso do estabelecimento das Melastomataceae de regiões de cerrado. Ali, elas permanecem por longos períodos no banco de sementes do solo e podem germinar quando recebem luz, mesmo por períodos curtos, apesar das variações diurnas de temperatura.

Palavras-chave: germinabilidade, luz, Melastomataceae, temperatura

\section{Introdução}

Estudos sobre a germinação de sementes são de grande valia para a compreensão das etapas do estabelecimento de uma comunidade vegetal, bem como de sua sobrevivência e regeneração natural
(Vázquez-Yanes \& Orozco-Segovia 1993). A influência de fatores ambientais, como luz e temperatura, na germinação de sementes é essencial para entender os aspectos ecofisiológicos e bioquímicos envolvidos nesse processo (Baskin \& Baskin 2001, Gomes et al. 2001, Ranieri et al. 2003).

1. Instituto de Botânica, Caixa Postal 3005, 01061-970 São Paulo, SP, Brasil

2. Autor para correspondência: rcarreira01@yahoo.com.br 
Em ambientes naturais as sementes podem ser encontradas sob diversas condições de luz e de temperatura, condições essas que podem variar de acordo com a estrutura do dossel (Lindig-Cisneiros $\&$ Zedler 2001). A percepção da qualidade da luz pelas plantas ocorre através do fitocromo que corresponde a uma classe de pigmentos fotorreceptores. O modo de ação desses pigmentos depende do tipo de radiação incidente, pois luz com alta relação vermelho/vermelhoextremo (V/VE) induz a forma ativa (FVe), promovendo a germinação de sementes fotossensíveis, enquanto sob luz com baixa relação $\mathrm{V} / \mathrm{VE}$, o fitocromo torna-se inativo (FV), inibindo a germinação (Smith 2000, Takaki 2001).

A resposta das sementes à luz pode controlar o tempo de germinação no campo, sendo um fator decisivo para a sobrevivência das plântulas (Válio \& Scarpa 2001). A temperatura afeta tanto a porcentagem como a velocidade de germinação por interferir na embebição e nos processos metabólicos (Castro \& Hilhorst 2004). Para cada população de sementes há uma faixa característica de temperatura na qual a germinação ocorre (Bewley \& Black 1994).

Dentre as famílias encontradas em bancos de sementes no solo de Cerrado da Reserva Biológica e Estação Experimental de Mogi Guaçu (RBEE de Mogi Guaçu), destacaram-se em termos quantitativos, as Melastomataceae (Sassaki et al. 1999, Cesarino 2002). Espécies do gênero Miconia são freqüentes em todas as fisionomias de cerrado da RBEE de Mogi Guaçu, de acordo com Mantovani (1987). Miconia albicans (Sw.) Triana é uma espécie arbustiva com até 2,5 $\mathrm{m}$ de altura. As flores, com pétalas brancas e odor imperceptível, agrupam-se em inflorescências e, entre novembro e março, ocorre a frutificação. $\mathrm{O}$ fruto é vermelho quando jovem e torna-se verde-jade quando maduro, com número de sementes variável (Martins et al. 1996). M. langsdorffii Cogn. é um arbusto com até três metros de altura, possui flores brancas e frutos atro-purpúreos com cerca de 15 sementes. A floração ocorre entre outubro e dezembro e o pico de frutificação ocorre em janeiro e fevereiro (Goldenberg \& Shepherd 1998). A espécie arbustiva M. stenostachya Schr. \& Mart. ex DC. floresce entre setembro e novembro e a frutificação se dá de novembro até início de fevereiro; sua flores são brancas e os frutos de cor atro-purpúrea contêm cerca de 30 sementes (Goldenberg \& Shepherd 1998). Miconia rubiginosa (Bonpl.) DC. é uma espécie arbórea, podendo atingir até seis metros de altura. A floração acontece entre outubro e dezembro; as suas flores são brancas com odor forte e doce e a frutificação ocorre nos meses de fevereiro a julho. Os frutos são violáceos e contêm poucas sementes (Goldenberg \& Shepherd 1998). Schizocentron elegans Meissn. é herbácea perene, com flores solitárias, esparsas, são formadas continuamente durante quase todo o ano. Já a espécie herbácea Tibouchina gracilis (Bonpl.) Cogn. floresce de outubro até junho e apresenta flores de coloração rósea-purpúreas até violáceas.

De modo geral, as plantas da família Melastomataceae produzem sementes pequenas, menores que $0,5 \mathrm{~mm}$; os cotilédones são foliáceos, praticamente sem reservas para garantir o início do desenvolvimento das plantas jovens (Carreira \& Zaidan 2003). Considerando a quantidade de sementes produzidas, a abundância de indivíduos dessa família na RBEE de Mogi Guaçu e a relativa facilidade com que germinam, é de interesse conhecer as condições de luz e temperatura que favorecem a germinação, de modo a fornecer dados básicos sobre esse processo em sementes de algumas Melastomataceae do Cerrado.

\section{Material e métodos}

Frutos maduros, assim definidos por sua coloração, de Miconia albicans, Miconia rubiginosa e $M$. stenostachya foram colhidos na RBEE de Mogi Guaçu, SP (22 $18^{\circ}$ 'S e $\left.47^{\circ} 11^{\prime} \mathrm{W}\right)$; os frutos de $M$. langsdorffii foram colhidos na Estação Experimental de Itirapina, SP $\left(22^{\circ} 15^{\prime} \mathrm{S}\right.$ e $\left.47^{\circ} 37^{\circ} \mathrm{W}\right)$, e os frutos de Schizocentron elegans e Tibouchina gracilis foram colhidos em canteiro experimental da Seção de Fisiologia e Bioquímica de Plantas do Instituto

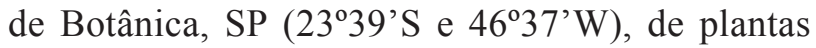
originadas de sementes colhidas na RBEE de Mogi Guaçu e cultivadas por três anos. Os frutos maduros foram colhidos de pelo menos oito indivíduos diferentes de cada espécie. Após a colheita, os frutos foram secados à temperatura ambiente do laboratório (cerca de $25^{\circ} \mathrm{C}$ ), em bandejas plásticas, por sete dias. Os frutos foram então armazenados a $4{ }^{\circ} \mathrm{C}$, em frascos tampados de vidro âmbar. As sementes foram retiradas dos frutos imediatamente antes da instalação dos experimentos, que se iniciaram cerca de 30 dias após as coletas.

A germinação foi realizada em câmaras B.O.B. Fanem, sob luz branca fluorescente contínua "luz do dia" com irradiância de $437 \mu \mathrm{W} . \mathrm{cm}^{-2}$ na altura das 
placas (Ruggiero \& Zaidan 1997). Para cada experimento de germinação foram feitas quatro repetições, cada uma com 50 sementes, em placas de Petri, forradas com duas folhas de papel de filtro e saturadas com água destilada. Nos testes de duração dos períodos de luz, foram utilizadas 30 sementes em cada placa. A contagem das sementes germinadas foi feita diariamente, durante 30 dias. Considerou-se germinação a protrusão da radícula ( $\geq 2 \mathrm{~mm}$ ).

Os experimentos sobre o efeito do fotoequilíbrio do fitocromo $(\mathrm{V}: \mathrm{VE}, \varphi)$ foram realizados no Laboratório de Fotomorfogênese de Plantas do Departamento de Botânica da UNESP/Rio Claro, SP, apenas em sementes de $M$. albicans e M. rubiginosa, a $25^{\circ} \mathrm{C}$. Para a obtenção do gradiente de fotoequilíbrio, foram utilizadas duas fontes de luz, instaladas $70 \mathrm{~cm}$ acima de uma superfície plana e equiidistantes dois metros, sobre a qual foram colocadas as placas de Petri contendo as sementes embebidas. Uma fonte de luz com alta relação V:VE (luz proveniente de lâmpadas fluorescentes "luz do dia", filtrada por uma placa de acrílico vermelho) e outra fonte de luz em posição oposta, com baixa relação $\mathrm{V}$ :VE (luz proveniente de lâmpadas incandescentes, filtrada através de uma placa de acrílico azul e uma placa vermelha sobrepostas), conforme Amaral-Baroli \& Takaki (2001). Cinco faixas para a disposição das placas foram demarcadas ao longo da superfície plana, para permitir a emissão dos valores teóricos de j: 0,564, $0,283,0,105,0,046$, e 0,099 , medidos com espectroradiômetro LI-1800 (LI-COR Instruments, Lincoln, Nebraska, USA) e determinados conforme Mancinelli (1994). Sementes embebidas no escuro atuaram como controle. A observação das sementes se deu sob luz verde de segurança, como indicado por Amaral-Baroli \& Takaki (2001).

Para avaliação da quantidade mínima de luz diária exigida pelas sementes, testes de germinação foram conduzidos sob diferentes períodos de luz: $0 \mathrm{~min}$ (escuro contínuo), $10 \mathrm{~min}, 15 \mathrm{~min}, 30 \mathrm{~min}, 60 \mathrm{~min}$, $90 \mathrm{~min}, 180 \mathrm{~min}, 360 \mathrm{~min}, 720 \mathrm{~min}$ e $1.440 \mathrm{~min}$ (luz contínua), a $25^{\circ} \mathrm{C}$, durante 30 dias. No tratamento de 720 min, uma câmara B.O.D. foi ajustada para manter as lâmpadas acesas apenas durante esse período; nos demais tratamentos, as placas contendo as sementes foram envolvidas por sacos plásticos pretos e expostas à luz apenas pelo período desejado. Em $M$. langsdorffii, os testes foram realizados nos tempos de exposição à luz de 0,90, 180, 360, 720 e 1.440 minutos, em função da disponibilidade de sementes.
A germinação das sementes foi testada sob temperaturas constantes de $15^{\circ} \mathrm{C}, 20^{\circ} \mathrm{C}, 25^{\circ} \mathrm{C}, 30^{\circ} \mathrm{C}$ e $35^{\circ} \mathrm{C}$ e alternadas de $30 / 20^{\circ} \mathrm{C}$, em câmaras B.O.D. Fanem, sob luz e escuro contínuos, conforme descrito anteriormente. Em sementes de $M$. langsdorffii, a germinação foi testada a $20^{\circ} \mathrm{C}, 25^{\circ} \mathrm{C}, 30^{\circ} \mathrm{C}$ e $30 / 20{ }^{\circ} \mathrm{C}$, devido à disponibilidade de sementes.

Foi utilizado o delineamento experimental inteiramente casualizado. As seguintes variáveis foram avaliadas: germinabilidade (G) e velocidade média (VM) da germinação. A velocidade média foi calculada de acordo com Labouriau \& Agudo (1987). Os dados de germinabilidade foram transformados em arco seno (\%) 0,5 antes de proceder à análise estatística. Os dados foram submetidos à análise de variância, utilizando-se o programa ANOVA, fator único, sendo adotado o nível de 5\% de significância.

\section{Resultados}

Em sementes de $M$. stenostachya e M. albicans, exposições diárias à luz por 15 e $60 \mathrm{~min}$, respectivamente, são suficientes para provocar germinação igual à obtida no tratamento com luz constante, não se constatando diferenças significativas entre os diferentes períodos de luz. Em sementes de M. albicans submetidas a períodos de luz de 10, 15 e 30 min, verificou-se uma redução significativa da germinação (figura 1). A germinabilidade ficou em torno de $50 \%$, sem diferenças significativas entre os períodos de luz fornecidos. As sementes de M. rubiginosa mostraram baixa germinabilidade, mesmo sob luz contínua (figura 1).

As sementes de $S$. elegans apresentaram germinabilidade ao redor de $50 \%$ sob períodos diários de luz de $60 \mathrm{~min}, 720 \mathrm{~min}$ e $1.440 \mathrm{~min}$ (figura 1). Entre 90 e 360 min diários de luz ocorreu redução significativa da germinação em relação ao valor obtido com 60 min diários de luz. A partir de 180 min diários de luz, as sementes de $T$. gracilis apresentaram germinação semelhante ao tratamento de luz contínua, ao redor de $60 \%$ (figura 1 ). Nenhuma das sementes das espécies estudadas germinou no escuro.

A velocidade média de germinação das sementes de $M$. albicans, M. langsdorffii e $M$. stenostachya foi superior sob períodos diários de luz de $1.440 \mathrm{~min}$, diferindo significativamente dos demais tratamentos (tabela 1). Nessas sementes, a velocidade média de germinação diminuiu com o tempo de exposição à luz. As sementes de $M$. rubiginosa, T. gracilis e 
S. elegans apresentaram velocidades maiores sob 10 min diários de luz (tabela 1).

As sementes de M. albicans germinaram em todos os gradientes de fotoequilíbrio do fitocromo fornecidos. De modo geral, à medida que o gradiente do fotoequilíbrio $(\varphi)$ aumenta, ocorreu também um aumento na germinabilidade das sementes. Verificou-se que as duas maiores razões $\mathrm{V}$ : $\operatorname{VE}(\varphi=0,564$

Miconia albicans

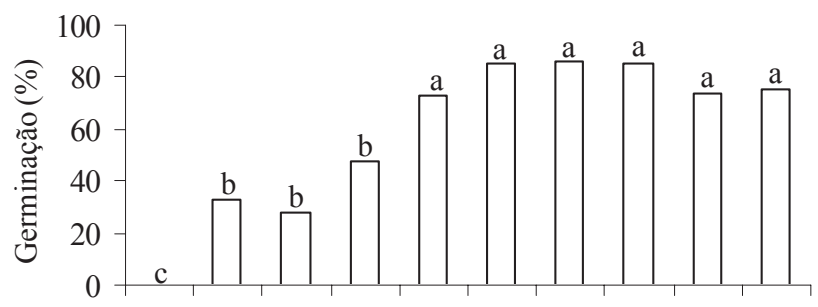

$\begin{array}{llllllllll}0 & 10 & 15 & 30 & 60 & 90 & 180 & 360 & 720 & 1440\end{array}$

\section{Miconia langsdorffii}

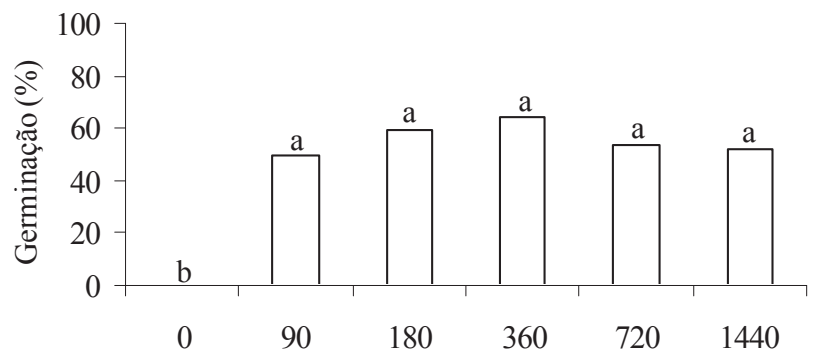

Miconia rubiginosa

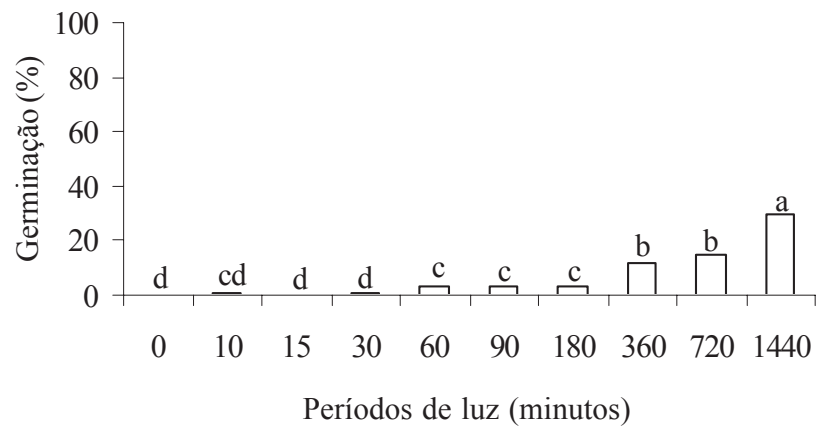

e 0,426$)$ promoveram significativamente a germinação (figura 2). Um decréscimo acentuado na porcentagem de germinação foi observado $\operatorname{com} \varphi=$ 0,228 . As sementes de $M$. rubiginosa mantiveram baixa germinabilidade em todos os gradientes de fotoequilíbrio testados, inclusive na maior razão $\mathrm{V}: \mathrm{VE}$ ( $8 \%$ de germinação, com diferença significativa em relação aos outros valores de $\varphi$ ) (figura 2).
Miconia 'stenostachya

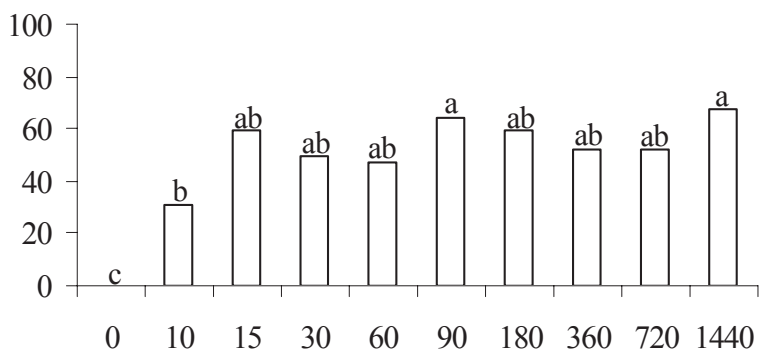

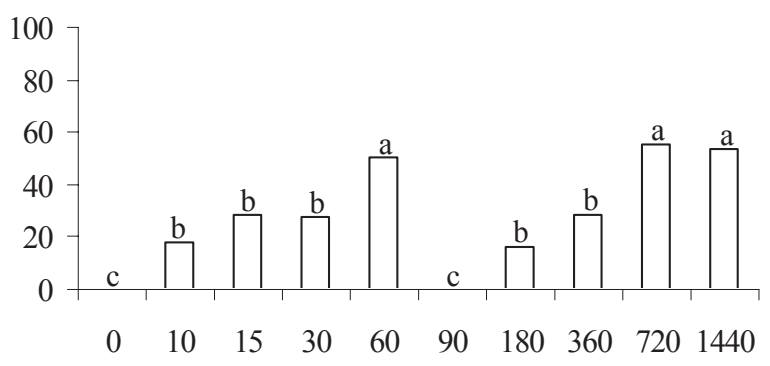

Tibouchina gracilis

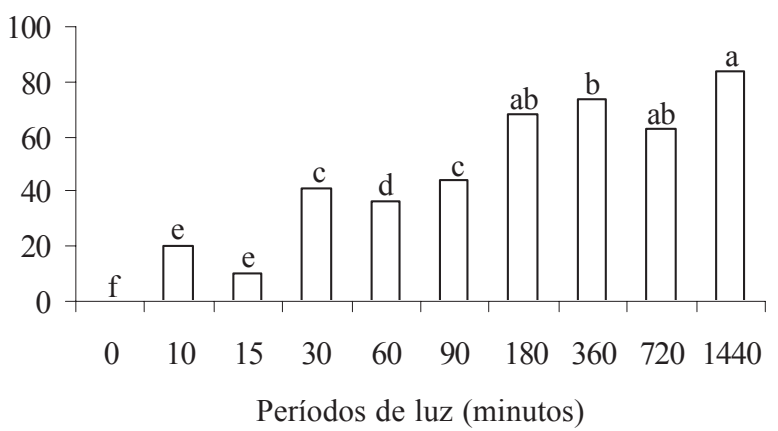

Figura 1. Germinação de sementes de Miconia albicans, M. langsdorffii, M. rubiginosa, M. stenostachya, Schizocentron elegans e Tibouchina gracilis à temperatura de $25^{\circ} \mathrm{C}, 30$ dias após o início da embebição, em períodos diários de luz de 0 (escuro contínuo), 10 min, $15 \mathrm{~min}, 30 \mathrm{~min}, 60 \mathrm{~min}, 90 \mathrm{~min}, 180 \mathrm{~min}, 360 \mathrm{~min}, 720 \mathrm{~min}$ e $1.440 \mathrm{~min}$ (luz contínua). Letras comparam a germinação entre os períodos de luz, para cada espécie. 
Tabela 1. Velocidade média de germinação de sementes de Miconia albicans (M alb), M. langsdorffii (M lang), M. rubiginosa (M rub), M. stenostachya (M steno), Schizocentron elegans (S eleg) e Tibouchina gracilis ( T gra) à temperatura de $25^{\circ} \mathrm{C}, 30$ dias após o início da embebição, em períodos diários de luz de 0 (escuro contínuo), 10 min, 15 min, 30 min, 60 min, 90 min, 180 min, 360 min, 720 min e 1440 min (luz contínua). Letras comparam cada medida entre os períodos de luz, para cada espécie.

\begin{tabular}{|c|c|c|c|c|c|c|}
\hline Períodos de luz & $\mathrm{M}$ alb & M lang & $\mathrm{M}$ rub & M sten & S eleg & T gra \\
\hline 0 (escuro) & - & - & - & - & - & - \\
\hline $10 \mathrm{~min}$ & $0,06 \mathrm{bc}$ & \# & $0,10 \mathrm{a}$ & $0,07 \mathrm{~cd}$ & $0,07 \mathrm{a}$ & $0,08 \mathrm{a}$ \\
\hline $15 \mathrm{~min}$ & $0,07 \mathrm{~b}$ & \# & - & $0,07 \mathrm{~cd}$ & $0,08 \mathrm{a}$ & $0,08 \mathrm{a}$ \\
\hline $30 \mathrm{~min}$ & $0,05 \mathrm{c}$ & \# & $0,05 \mathrm{~b}$ & $0,06 \mathrm{~d}$ & $0,09 \mathrm{a}$ & $0,04 \mathrm{c}$ \\
\hline $60 \mathrm{~min}$ & $0,09 \mathrm{~b}$ & \# & $0,03 \mathrm{~b}$ & $0,07 \mathrm{~cd}$ & $0,06 \mathrm{ab}$ & $0,06 \mathrm{~b}$ \\
\hline $90 \mathrm{~min}$ & $0,08 \mathrm{~b}$ & $0,04 \mathrm{~b}$ & $0,02 \mathrm{~b}$ & $0,09 \mathrm{bc}$ & - & $0,05 \mathrm{bc}$ \\
\hline $180 \mathrm{~min}$ & $0,09 \mathrm{~b}$ & $0,04 \mathrm{~b}$ & $0,03 \mathrm{~b}$ & $0,10 \mathrm{bc}$ & $0,04 \mathrm{~b}$ & $0,06 \mathrm{~b}$ \\
\hline $360 \mathrm{~min}$ & $0,10 \mathrm{ab}$ & $0,04 \mathrm{~b}$ & $0,04 \mathrm{~b}$ & $0,09 \mathrm{bc}$ & $0,04 \mathrm{~b}$ & $0,07 \mathrm{ab}$ \\
\hline $720 \mathrm{~min}$ & $0,10 \mathrm{ab}$ & $0,04 \mathrm{~b}$ & $0,04 \mathrm{~b}$ & $0,11 \mathrm{ab}$ & $0,05 \mathrm{~b}$ & $0,07 \mathrm{ab}$ \\
\hline $1440 \mathrm{~min}$ & $0,13 \mathrm{a}$ & $0,05 \mathrm{a}$ & $0,05 \mathrm{~b}$ & $0,14 \mathrm{a}$ & $0,06 a b$ & $0,07 \mathrm{ab}$ \\
\hline
\end{tabular}

-: não calculado; \#: não realizado

A figura 3 mostra os dados de germinabilidade e velocidade média de germinação sob diferentes temperaturas, nas espécies estudadas. Com relação ao efeito da temperatura na germinação, as sementes de $M$. albicans e $M$. stenostachya não apresentaram diferenças significativas em termos de germinabilidade sob as temperaturas constantes de 20,25 e $30^{\circ} \mathrm{C}$ e alternadas de $30 / 20^{\circ} \mathrm{C}$, ao final de 30 dias (figura 3 ). A $15^{\circ} \mathrm{C}$ e a $35^{\circ} \mathrm{C}$ houve uma diminuição significativa da germinação e da velocidade. As sementes de $M$. albicans germinaram mais rapidamente a $25^{\circ} \mathrm{C}$ e $30^{\circ} \mathrm{C}$ e as de $M$. stenostachya, a $25^{\circ} \mathrm{C}, 30^{\circ} \mathrm{C}$ e $30 / 20^{\circ} \mathrm{C}$. A germinação das sementes de $M$. langsdorffii foi

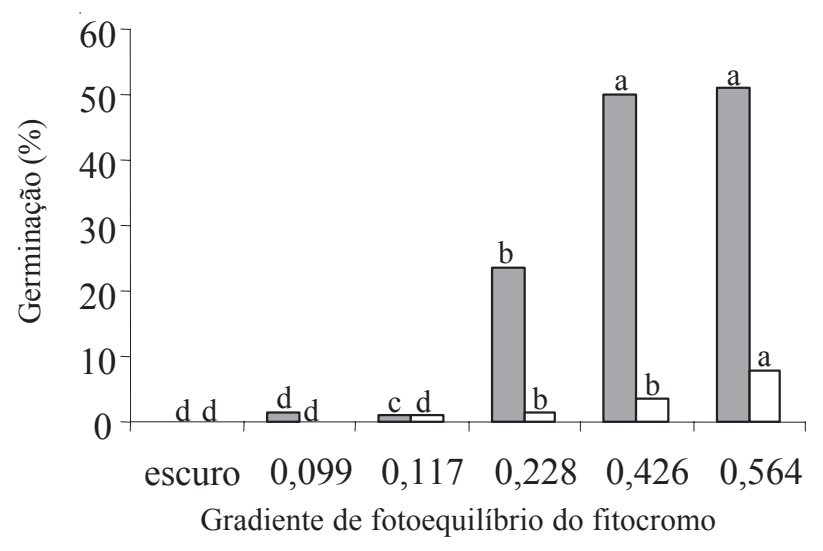

Figura 2. Efeito do gradiente de fotoequilíbrio do fitocromo $(\varphi)$ sobre a germinação de sementes de Miconia albicans e M. rubiginosa. Letras comparam a germinação entre os fotoequilíbrios, após 30 dias do início da embebição, em cada espécie. $\square=$ Miconia albicans; $\square=$ Miconia rubiginosa . semelhante a $25^{\circ} \mathrm{C}$ e $30 / 20^{\circ} \mathrm{C}$, porém as sementes germinaram mais rapidamente a $25^{\circ} \mathrm{C}$. As sementes de $M$. rubiginosa mostraram baixa germinabilidade em todas as temperaturas e não germinaram a $15^{\circ} \mathrm{C}$ (figura 3) e as temperaturas que mais favoreceram a velocidade de germinação foram $25^{\circ} \mathrm{C}$ e $35^{\circ} \mathrm{C}$.

Sementes de $T$. gracilis germinaram melhor a $25{ }^{\circ} \mathrm{C}, 30{ }^{\circ} \mathrm{C}$ e $30 / 20{ }^{\circ} \mathrm{C}$ sem diferença significativa entre as temperaturas. A $30{ }^{\circ} \mathrm{C}$ foi obtida germinabilidade significativamente superior às demais temperaturas com sementes de $S$. elegans (figura 3). Essas sementes não germinaram a $15{ }^{\circ} \mathrm{C}$ e as de T. gracilis germinaram significativamente menos a $15^{\circ} \mathrm{C}, 20{ }^{\circ} \mathrm{C}$ e $35^{\circ} \mathrm{C}$. A velocidade média de germinação em sementes de $S$. elegans foi favorecida pelas temperaturas de $25^{\circ} \mathrm{C}, 30^{\circ} \mathrm{C}$ e $30 / 20^{\circ} \mathrm{C}$ e em T. gracilis, apenas em $25^{\circ} \mathrm{C}$.

\section{Discussão}

As sementes das melastomatáceas de Cerrado aqui estudadas são fotoblásticas e apresentaram plasticidade de resposta de germinação em relação à luz. Quanto ao período de luz necessário para desencadear o processo de germinação, M. albicans, $M$. langsdorffii, S. elegans e $T$. gracilis exigem entre 60 e 90 min diários de luz para dar uma resposta de germinação semelhante à obtida sob luz contínua. M. stenostachya (15 min) e M. rubiginosa (360 min) seriam os extremos desse tipo de comportamento, entre as espécies estudadas, nas condições experimentais. 
De acordo com a literatura, as espécies de Melastomataceae ocorrentes em Mata Atlântica têm sua germinação ao redor de $30 \%$ e o processo é desencadeado quando são fornecidas, no mínimo, $120 \mathrm{~min}$ diários de luz (Amaral \& Paulilo 1991/1992, Leite 1998, Zaia \& Takaki 1998, Freitas 1998, Brischi 2000, Godoi 2002 e Simão 2005). De maneira geral, o aumento do período de luz fornecido, promove a germinação.
Miconia albicans

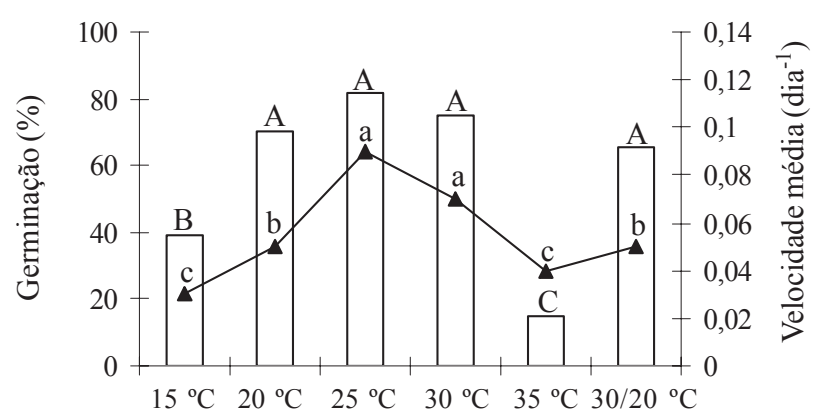

Miconia langsdorffii

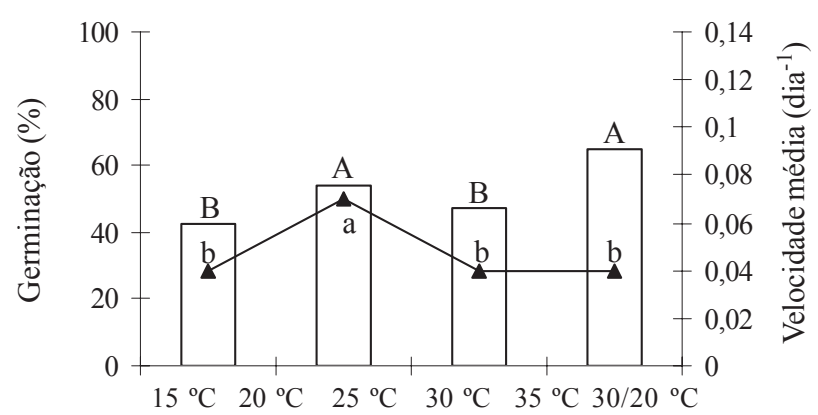

Miconia rubiginosa

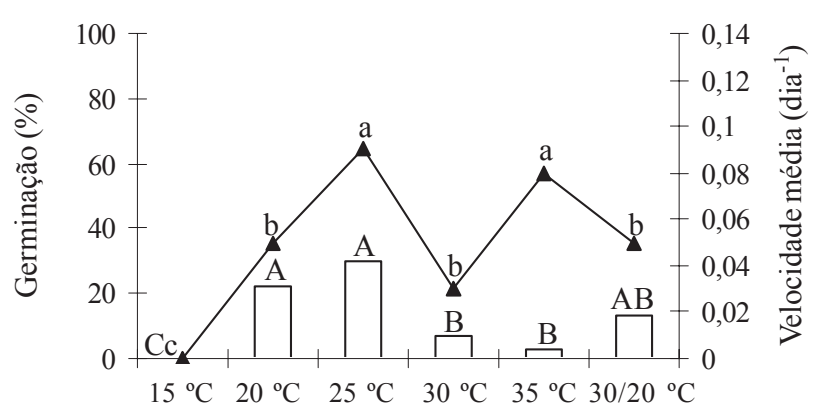

Temperatura de germinação
Miconia stenostachya

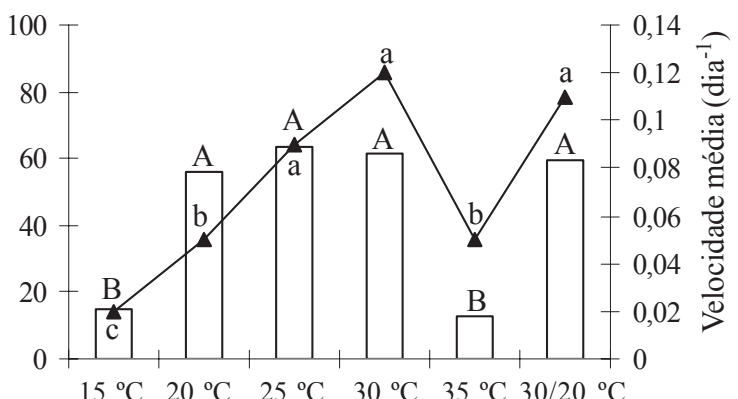

Schizocentron elegans

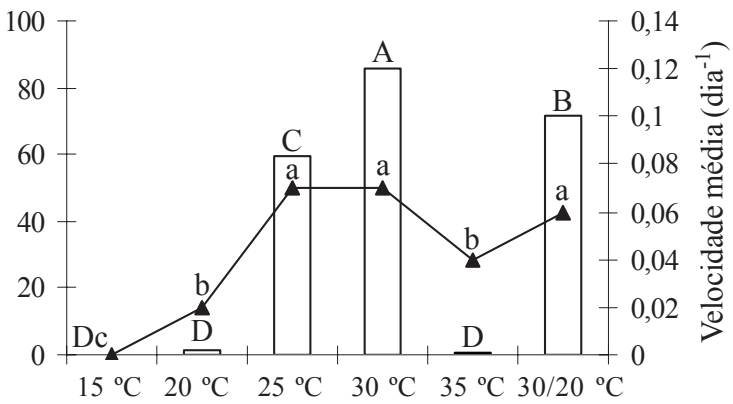

Tibouchina gracilis

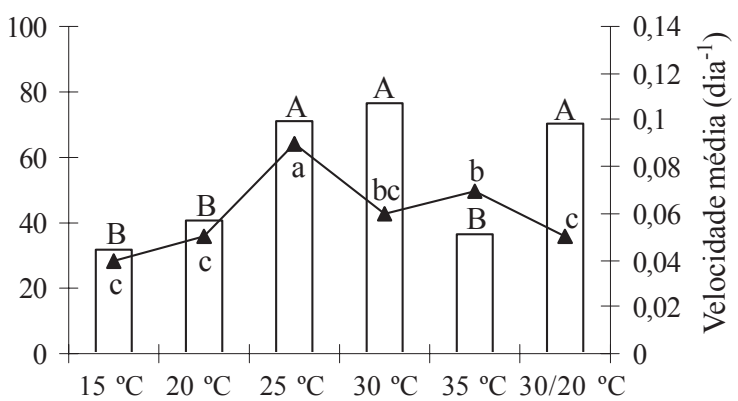

Temperatura de germinação

Figura 3. Germinabilidade e velocidade média de germinação de sementes de Miconia albicans, M. langsdorffii, M. rubiginosa, M. stenostachya, Schizocentron elegans e Tibouchina gracilis em temperaturas constantes de $15{ }^{\circ} \mathrm{C}, 20^{\circ} \mathrm{C}, 25^{\circ} \mathrm{C}, 30^{\circ} \mathrm{C}$ e $35{ }^{\circ} \mathrm{C}$ e temperaturas alternadas de $30 / 20^{\circ} \mathrm{C}$, sob luz constante. Letras maiúsculas comparam os dados finais de germinação entre as temperaturas, em cada espécie; letras minúsculas comparam a velocidade média de germinação das sementes entre as temperaturas. $\square=$ Germinação; - - - = Velocidade 
A indução da germinação está relacionada com a presença ou ausência de luz, quantidade e a qualidade da luz (Kendrick \& Frankland 1983). O papel do fitocromo em sementes tem sido relacionado com a manutenção da dormência em sementes que se encontram enterradas nas camadas superficiais do solo. Possivelmente, essas sementes respondem às mudanças de temperatura, da razão $\mathrm{V}: \mathrm{VE}$, da duração do período de luz e do controle ambiental da qualidade da luz (Casal \& Sánchez 1998). O aumento da razão $\mathrm{V}$ :VE promoveu um aumento na porcentagem final de germinação das sementes de duas espécies aqui testadas, M. albicans e M. rubiginosa, apesar da germinabilidade desta última ter sido muito baixa, talvez devido à variação do lote de sementes utilizadas.

$\mathrm{O}$ estudo do comportamento germinativo de sementes frente a diferentes gradientes de fotoequilíbrio do fitocromo é importante no sentido de conhecer o grau de tolerância das espécies às diferentes razões $\mathrm{V}$ :VE que podem ocorrer em ambientes naturais (Válio \& Scarpa 2001). Assim, podem ser feitas escolhas mais seguras quanto às espécies a serem utilizadas em projetos de reflorestamento a partir de semeadura direta, como comentado por Barbosa et al. (1992, 1996). A presença de sementes fotoblásticas é bem conhecida na família Melastomataceae (Andrade 1995, Zaia \& Takaki 1998, Baskin et al. 1999, Válio \& Scarpa 2001). Sementes de espécies desta família que habitam ambientes de dossel fechado geralmente são pequenas e podem permanecer no solo à espera da abertura de clareiras que proporcionam a entrada de luz (Silveira et al. 2004). Outras espécies de Melastomataceae de Mata Atlântica demonstraram possuir padrão de germinação semelhante em relação à razão V:VE (Leite 1998, Zaia \& Takaki 1998, Freitas 1998, Brischi 2000, Godoi 2002, Simão 2005). O presente trabalho relata os primeiros resultados do efeito da relação V/VE em melastomatáceas de Cerrado.

Quanto ao efeito da temperatura, a maior germinabilidade nas sementes das Melastomataceae de Cerrado foi obtida nas temperaturas entre $20^{\circ} \mathrm{C}$ e $30{ }^{\circ} \mathrm{C}$, com alterações na velocidade média de germinação. As temperaturas de $15^{\circ} \mathrm{C}$ e $35^{\circ} \mathrm{C}$ afetaram negativamente a germinação das sementes, corroborando dados de que temperaturas entre $20{ }^{\circ} \mathrm{C}$ e $30^{\circ} \mathrm{C}$ são mais favoráveis para desencadear o processo germinativo (Melo et al. 1998). No entanto, foi observado que, as sementes que não germinaram, também embeberam e mantiveram-se firmes, não sendo encontrados traços de deterioração ou contaminação por microrganismos.

A germinação não ocorreu no escuro em nenhuma das temperaturas testadas (dados não mostrados). A alternância de temperaturas não encurtou o período para o início da germinação, nem a germinabilidade dessas sementes em relação a $25^{\circ} \mathrm{C}$. Mayer \& Poljakoff-Mayber (1989) relataram que temperaturas alternadas podem ser mais eficazes na promoção da germinação do que cada temperatura aplicada isoladamente, fato que não foi demonstrado nas sementes aqui estudadas, como também em Miconia chamissois Naudin (Válio \& Scarpa 2001), Tibouchina mutabilis Cogn. (Simão 2005), T. multiflora Cogn. (Garcia et al. 2006), Marcetia taxifolia (A. St.-Hil.) DC. (Silveira et al. 2004). Outro efeito de temperaturas alternadas na germinação é o de substituir o efeito da luz em sementes fotoblásticas, como em Bidens gardneri Baker (Sassaki et al. 1999), uma espécie herbácea também de Cerrado. Em nenhuma das espécies estudadas esse regime de temperatura reverteu o efeito do escuro (dados não mostrados); apenas a velocidade de germinação foi afetada pelas temperaturas alternadas, na luz. Sementes de M. cinnamomifolia (DC.) Naudin (Leite 1998) e de Huberia semiserrata DC. (Brischi 2000), espécies de Melastomataceae de Mata Atlântica, germinaram na ausência de luz, mas em valores inferiores a $5 \%$.

Tendo em vista que a germinação das sementes constitui o primeiro passo para a exploração de novos habitats, este processo pode influenciar, em termos quantitativos, as populações das espécies em suas áreas de ocorrência (Ramírez-Padilla \& Valverde 2005). Com base nos dados obtidos neste estudo e nos de diversos autores, é provável que a distribuição das espécies de Melastomataceae, nas regiões de Cerrado e de Mata Atlântica, seja influenciada principalmente pela quantidade e qualidade de luz disponíveis para as sementes. Secundariamente, a temperatura poderia afetar a germinação, provavelmente na velocidade do processo.

\section{Agradecimentos}

Ao Prof. Dr. Massanori Takaki, do Departamento de Botânica da UNESP/Rio Claro por permitir o uso das instalações do Laboratório de Fotomorfogênese de Plantas. À CAPES e ao CNPq, pela concessão das bolsas de Mestrado a R.C. Carreira e de produtividade a L.B.P. Zaidan. 


\section{Literatura citada}

Amaral, L.I.V. \& Paulilo, M.T.S. 1991/1992. Efeito da luz, temperatura, reguladores de crescimento e nitrato de potássio na germinação de Miconia cinnamomifolia (DC.) Naudin. Ínsula 21: 59-86.

Amaral-Baroli, A. \& Takaki, M. 2001. Phytochrome controls achene germination in Bidens pilosa L. (Asteraceae) by very low fluence response. Brazilian Archieves of Biology and Technology 44: 121-124.

Andrade, A.C.S. 1995. Efeito da luz e da temperatura na germinação de Leandra breviflora Cogn., Tibouchina benthamiana Cogn., Tibouchina grandiflora Cogn. e Tibouchina moricandiana (DC.) Baill. (Melastomataceae). Revista Brasileira de Sementes 17: 29-35.

Barbosa, J.M., Barbosa, L.M., Silva, T.S., Gatuzzo, E.H. \& Freire, R.M. 1992. Capacidade de estabelecimento de indivíduos de espécies da sucessão secundária a partir de sementes em sub-bosque de uma mata ciliar degradada do rio Moji-Guaçú/SP. In: V.L. Mattes, J.L. Grossi, J. Skalok Júnior, J.H. Faraco Júnior \& H.L.A. Telles (eds.). Anais do I Simpósio Nacional de Recuperação de Áreas Degradadas, Curitiba, pp. 400-406.

Barbosa, J.M., Santos, M.R.O., Psiotano, W.A., Barbosa, L.M. \& Santos, S.R.G. 1996. Estabelecimento de indivíduos de Inga uruguensis Hook. et Arn. a partir do plantio de sementes em uma área ciliar degradada, considerando diferentes condições de luz e umidade do solo. In: M. Balensiefer, A.J. Araujo \& N.C. Rosot (eds.). Anais do IV Simpósio Internacional sobre Ecossistemas Florestais, Belo Horizonte, pp. 191-193.

Baskin, C.C. \& Baskin, J.M. 2001. Seeds: Ecology, Biogeography, and Evolution of Dormancy and Germination. Academic Press, San Diego.

Baskin, C.C., Baskin, J.M. \& Chester-Edward, W. 1999. Seed dormancy and germination in Rhexia mariana var. interior (Melastomataceae) and ecoevolutionary implications. Canadian Journal of Botany 77: 488-493.

Bewley, J.D. \& Black, M. 1994. Seeds: physiology of development and germination. 2 ed. Plenum Press, New York.

Brischi, A.M. 2000. Efeito da luz e da temperatura na germinação de sementes de Huberia semiserrata DC. (Melastomataceae). Dissertação de Mestrado, Universidade Estadual Paulista, Rio Claro.

Carreira, R.C. \& Zaidan, L.B.P. 2003. Estabelecimento e crescimento inicial de Miconia albicans (Sw.) Triana e Schizocentron elegans Meissn., sob fotoperíodos controlados. Hoehnea 30: 155-161.

Casal, J.J. \& Sánchez, R.A. 1998. Phytochromes and seed germination. Seed Science Research 8: 317-329.
Castro, R.D. \& Hilhorst, H.W.M. 2004. Embebição e reativação do metabolismo. In: A.G. Ferreira \& F. Borghetti (eds.). Germinação: do básico ao aplicado. Artmed, Porto Alegre, pp.149-162.

Cesarino, F. 2002. Bancos de sementes do solo da Reserva Biológica e Estação Experimental de Moji-Guaçu, em área de cerrado no Estado de São Paulo. Tese de Doutorado, Universidade Estadual de Campinas, Campinas.

Freitas, N.P. 1998. Análise do efeito da luz na germinação de sementes de Tibouchina pulchra Cogn. e Tibouchina sellowiana (Melastomataceae). Tese de Doutorado, Universidade Estadual Paulista, Rio Claro.

Garcia, L.C., Barros, F.V. \& Lemos Filho, J.P. 2006. Comportamento germinativo de duas espécies de canga ferrífera: Baccharis retusa DC. (Asteraceae) e Tibouchina multiflora Cogn. (Melastomataceae). Acta Botanica Brasilica 20: 443-448.

Godoi, S. 2002. Aspectos ecofisiológicos da germinação de sementes de Miconia theaezans (Bonpl.) Cogniaux (Melastomataceae). Tese de Doutorado, Universidade Estadual Paulista, Rio Claro.

Goldenberg, R. \& Shepherd, G.J. 1998. Studies on the reprodutive biology of Melastomataceae in "cerrado" vegetation. Plant Systematics and Evolution 211: 13-29.

Gomes, V., Madeira, J.A., Fernandes, G.W. \& Lemos Filho, J.P. 2001. Seed dormancy and germination of sympatric species of Chamaecrista (Leguminosae) in a rupestrian field. International Journal of Ecology and Environmental Sciences 27: 191-197.

Kendrick, R.E. \& Frankland, B. 1983. Phytochrome and plant growth. The Camelot Press, Southampton.

Labouriau, L.G. \& Agudo, M. 1987. On the physiology of seed germination in S. hispanica L.I. Temperature effects. Anais da Academia Brasileira de Ciências 59: 37-56.

Leite, I.T.A. 1998. Aspectos fisioecológicos da germinação de sementes de Miconia cinnamomifolia (D.C.) Naud. - Melastomataceae. Tese de Doutorado, Universidade Estadual Paulista, Rio Claro.

Lindig-Cisneiros, R. \& Zedler, J. 2001. Effects of light on seed germination in Aphalaris arundinaceae L. (reed canary grass). Plant Ecology 155: 75-78.

Mancinelli, A.L. 1994. The physiology of phytochrome action. In: R.E. Kendrick \& G.H.M. Kronemberg (eds.). Photomorphogenesis in plants. 2ed. The Netherlands: Kluwer Academic Publishers, Dordrecht, pp. 211-269.

Mantovani, W. 1987. Análise florística e fitossociológica do estrato herbáceo-subarbustivo do cerrado na Reserva Biológica de Moji Guaçu e em Itirapina, SP. Tese de Doutorado, Universidade Estadual de Campinas, Campinas.

Martins, A.B., Semir, J., Goldenberg, R. \& Martins, E. 1996. O Gênero Miconia Ruiz \& Pav. Melastomataceae no Estado de São Paulo. Acta Botanica Brasilica 10: 267-314. 
Mayer, A.M. \& Poljakoff-Mayber, A. 1989. The germination of seeds. Pergamon Press, Oxford.

Melo, J.T., Silva, J.A., Torres, R.A.A., Silveira, C.E.S \& Caldas, L.S.C. 1998. Coleta, propagação e desenvolvimento inicial de espécies do cerrado. In: S.M. Sano \& S.P. Almeida (eds.) Cerrado: ambiente e flora. Embrapa. Planaltina, D.F., pp. 195-243.

Ramírez-Padilla, C.A. \& Valverde, L. 2005. Germination responses of three cogeneric cacus species (Neobuxbaumia) with differents degrees of rarity. Journal of Aris Environments 61: 333-343.

Ranieri, B.D., Lana, T.C., Negreiros, D., Araújo, L.M. \& Fernandes, G.W. 2003. Germinação de sementes de Lavoisiera cordata Cogn. e Lavoisiera francavillana Cogn. (Melastomataceae), espécies simpátricas da Serra do Cipó, Brasil. Acta Botanica Brasilica 17: 523-530.

Ruggiero, P.G.C. \& Zaidan, L.B.P. 1997. Estudos de desenvolvimento de Viguiera robusta Gardn., uma Asteraceae do cerrado. Revista Brasileira de Botânica 20: $1-9$.

Sassaki, R.M., Zaidan, L.B.P. \& Felippe, G.M. 1999. Effect of storage of achenes of Bidens gardneri Baker on light sensitivity during germination. Revista Brasileira de Botânica 22: 75-81.
Silveira, F.A.O., Negreiros, D. \& Fernandes, G.W. 2004. Influência da luz e da temperatura na germinação de sementes de Marcetia taxifolia (A. St.-Hil.) DC. (Melastomataceae). Acta Botanica Brasílica 18: 847-851.

Simão, E. 2005. Estudo da germinação de sementes de Tibouchina mutabilis (Vell.) Cogn. (Melastomataceae). Dissertação de Mestrado, Universidade Estadual Paulista, Rio Claro.

Smith, H. 2000. Phytochromes and light signal perception by plants - an emerging synthesis. Nature 407: 585-591.

Takaki, M. 2001. New proposal of classification of seeds based on forms of phytochrome instead of photoblastism. Revista Brasileira de Fisiologia Vegetal 13: 103-107.

Válio, I.F.M. \& Scarpa, F.M. 2001. Germination of seeds of tropical pioneer species under controlled and conditions natural. Revista Brasileira de Botânica 24: 79-84.

Vázquez-Yanes, C. \& Orozco-Segovia, A. 1993. Patterns of seed longevity and germination in tropical rain forest. Annual Review of Ecology and Systematics 24: 69-87.

Zaia, J.E. \& Takaki, M. 1998. Estudo da germinação de sementes de espécies arbóreas pioneiras: Tibouchina pulchra Cogn. e Tibouchina granulosa Cogn. (Melastomataceae). Acta Botanica Brasilica 12: 221-229. 